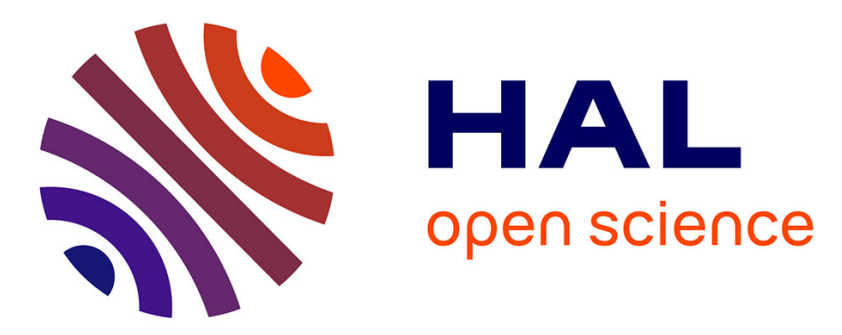

\title{
Résultats de mesures magnétiques sur l'équipement électronique du satellite HEOS-A1
}

\author{
A. Vuye
}

\section{To cite this version:}

A. Vuye. Résultats de mesures magnétiques sur l'équipement électronique du satellite HEOS-A1. Revue de Physique Appliquée, 1970, 5 (1), pp.59-71. 10.1051/rphysap:019700050105900 . jpa-00243376

\section{HAL Id: jpa-00243376 https://hal.science/jpa-00243376}

Submitted on 1 Jan 1970

HAL is a multi-disciplinary open access archive for the deposit and dissemination of scientific research documents, whether they are published or not. The documents may come from teaching and research institutions in France or abroad, or from public or private research centers.
L'archive ouverte pluridisciplinaire HAL, est destinée au dépôt et à la diffusion de documents scientifiques de niveau recherche, publiés ou non, émanant des établissements d'enseignement et de recherche français ou étrangers, des laboratoires publics ou privés. 


\title{
RÉSULTATS DE MESURES MAGNÉTIQUES SUR L'ÉQUIPEMENT ÉLEGTRONIQUE DU SATELLITE HEOS-A1
}

\author{
Par A. VUYE, \\ Société d'Études Techniques et Constructions Aérospatiales (E.T.G.A.), Charleroi (Belgique).
}

\begin{abstract}
Résumé. - Au début de l'année 1966, la Société E.T.C.A. a été chargée de la réalisation de sous-ensembles électroniques fondamentaux au fonctionnement du satellite HEOS-A1. Le faible niveau de champ magnétique résiduel propre à ce satellite à l'endroit du magnétomètre d'une expérience (S24A $\alpha$ de 1'Imperial College) constitue une des caractéristiques importantes du projet HEOS-A1. Le faible niveau magnétique requis $(1 \gamma$ à l'endroit de $S 24 A \alpha)$ pour tout le satellite a entraîné des contraintes spéciales sur chacun des sous-ensembles embarqués, électroniques ou non.

Il en est résulté un effort intensif au niveau de tout le projet dans les domaines d'étude, de moyens d'essais, d'essais proprement dits et de surveillance chez tous les contractants et spécialement pour les boîtiers d'électroniques.

Le présent exposé décrira brièvement :

- Les contraintes magnétiques d'HEOS-A1;

- Les moyens d'essais mis sur pied ou disponibles tout au long du projet;

- L'organisation de la section "magnétisme " dans un contexte élargi de fiabilité des équipements (pris globalement), et des essais d'environnements - incidence sur la fabrication ;

- Les essais effectués sur les boîtiers eux-mêmes et l'effort développé pour réduire le champ magnétique résiduel à un niveau aussi faible que possible ;

- Certains essais utilisant un appareillage plus spécialisé (magnétomètres à vapeur de rubidium) seront présentés et les résultats discutés.
\end{abstract}

Enfin, l'interaction au niveau du projet a stimulé des contacts débordant largement le cadre industriel du projet.

Introduction. - En novembre 1965, le Consortium Industriel :

Junkers Flugzeug und Motorenwerke $\mathrm{GmbH}$

$$
\begin{aligned}
& \text { (J.F.M., Germany) } \\
& \text { (Germany) } \\
& \text { (B.A.C., United King- } \\
& \text { dom) }
\end{aligned}
$$

Messerschmidt A.G.

Études Techniques et Constructions Aérospatiales S.A.

(E.T.C.A., Belgique)

Société Nationale d'Études et Construction de Moteurs d'Aviation

(S.N.E.G.M.A., France)

s'est vu confier, par l'E.S.R.O., l'étude, le développement, la réalisation et les essais du troisième satellite de cette organisation spatiale européenne.

Le satellite HEOS-A1 ( $f$ ig. 1) devait, en ordre principal, effectuer des mesures à l'extérieur de la magnétosphère et dans la zone de l'onde de choc.

E.T.C.A. a participé activement à l'élaboration du satellite par la fourniture de l'équipement électronique banalisé de bord, les équipements d'essais au sol (électronique), la botte du satellite (câblage interne), la participation aux essais et aux intégrations.

HEOS-A1 a été lancé, avec succès, le 5 décembre 1968 du Cap Kennedy. Tous les équipements de bord ont fonctionné normalement et les expériences ont fourni les premiers résultats scientifiques.

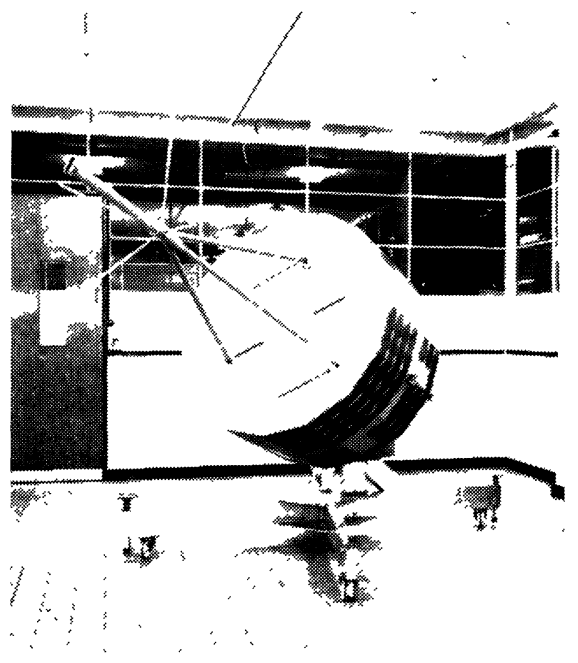

FIG. 1. - Le satellite HEOS-A1.

1. Missions et caractéristiques du satellite. 1.1. - HEOS-A1 (Highly Excentric Orbital Satellite) a été conçu pour embarquer huit expériences scientifiques. Celles-ci sont destinées à étudier le champ magnétique (à l'extérieur de la magnétosphère) et la distribution d'énergie de protons durant une période de grande activité solaire.

Son orbite est telle que l'apogée peut être vue de l'hémisphère nord, le périgée se trouvant dans l'hémisphère sud. L'apogée est de l'ordre de $250000 \mathrm{~km}$, 
le périgée de l'ordre de 200 à $300 \mathrm{~km}$ et la période orbitale est de l'ordre de 4,7 jours. Le poids total de ces expériences est de l'ordre de $25 \mathrm{~kg}$ et la puissance consommée $7,5 \mathrm{~W}$.

Cinq de ces expériences effectuent des mesures directionnelles, ce qui exige que :

- le satellite tourne sur lui-même à la vitesse de $10 \mathrm{rpm}$;

- le satellite soit capable de pointer son axe de rotation parallèlement ou perpendiculairement au plan de l'écliptique ou dans toute autre position intermédiaire;

- pendant douze mois (durée de vie du satellite), l'angle d'aspect solaire restera toujours compris entre 60 et $120^{\circ}$;

- l'attitude de l'axe de rotation du satellite soit connue avec une précision de $2^{\circ}$;

- le matériel de bord de référence d'attitude fournisse aux expérimentateurs les impulsions définissant l'orientation du satellite pour les expériences.

Deux expériences ont des exigences particulières qui ont influencé, d'une manière fondamentale, la conception générale du satellite :

- S24A requiert que le champ magnétique, produit par le satellite lui-même à l'endroit du magnétomètre de mesure, soit une petite fraction du champ magnétique interplanétaire (c'est-à-dire moins de $1 \gamma)$;

- S16 requiert qu'une capsule de $8 \mathrm{~kg}$ soit éjectée $\mathrm{du}$ satellite quelque temps après injection sur orbite.

1.2. Durée de vie. - La durée de vie du satellite doit être de 12 mois pendant lesquels la probabilité de panne catastrophique pour tous les états de fonctionnement doit être inférieure à $5 \%$ en excluant les expériences scientifiques et l'équipement de télécommunication.

2. Matériel fourni par E.T.C.A. dans le cadre du programme HEOS-A1. - La fourniture d'E.T.C.A. se situait principalement parmi les ensembles et sousensembles embarqués et le matériel d'essais au sol.

2.1. Sous-ensemble ÉLEGTRONIQUe d'Alimentation. - Le sous-ensemble électronique d'alimentation fournit les tensions électriques pour l'ensemble des équipements du satellite. L'énergie solaire collectée par les cellules photovoltaïques constitue la source primaire d'alimentation. Comme alimentation secondaire, une batterie argent-cadmium (magnétiquement propre) est utilisée en période de courte éclipse.

Le sous-ensemble électronique d'alimentation fournit la régulation, la conversion de tension, la surveillance et les commutations d'alimentation demandées par les expérimentateurs.

La puissance totale requise par le satellite est de $42 \mathrm{~W}$, y compris la puissance dissipée dans le soussystème d'alimentation.

Les communs d'alimentation. - Pour des raisons de propreté magnétique, les lignes d'alimentation sont flottantes par rapport à la structure. Le plus grand soin est pris pour éviter tout commun mis à la masse locale. Chaque expérimentateur est relié à l'alimentation par un ensemble de fils contenant le retour commun d'alimentation. Le seul « chemin de terre » de l'engin est connecté au travers du sous-système d'alimentation. Aucune « mise à la terre » de boîtier n'est tolérée, vu l'effet sur l'échange de courant à partir de cet équipement.

Bloc diagramme de l'alimentation. - Le bloc diagramme de l'alimentation est fourni par la figure 2 ci-après et montre la philosophie qui a été suivie pour la conception de celle-ci.

2.2. Sous-Système de tÉlÉGommunication. - Le système de télécommunication HEOS-A1 est un ensemble intégré du type répondeur servant simultanément aux 3 fonctions de télécommunication suivantes :

1. Télémesure.

2. Télécommande.

3. Tracking.

Dans ce sous-système, E.T.C.A. fournissait les antennes et les « baluns ». A cause du mouvement orbi-

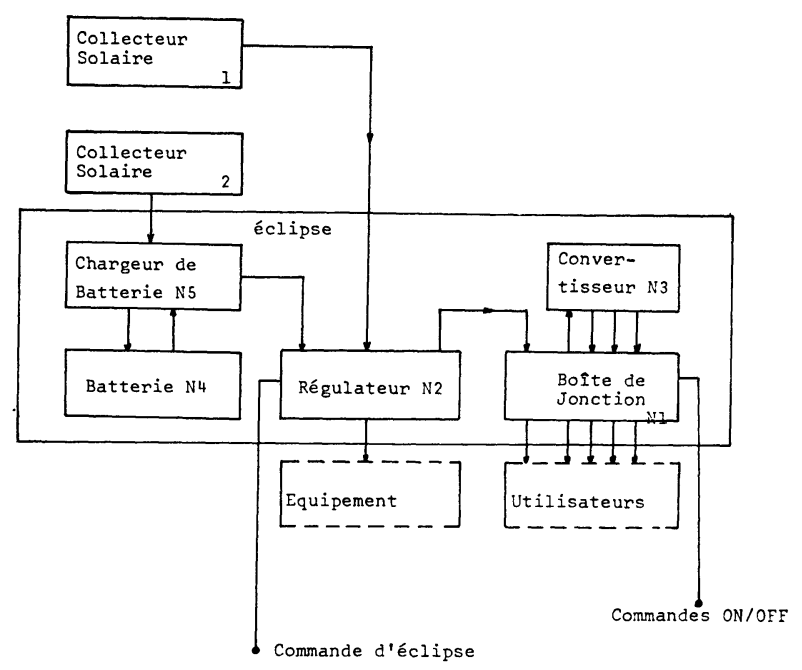

FIG. 2. - Bloc diagramme du système d'alimentation du satellite HEOS-A1.

tal et de la possibilité de réorientation du satellite (HEOS-A1 voit la terre sous différents angles) une antenne omnidirectionnelle est obligatoire.

Le diagramme d'antenne est isotropique avec des « trous » ne pouvant pas être supérieurs à $4 \mathrm{~dB}$.

L'antenne est constituée d'un " dipôle croisé », monté sur le mât du magnétomètre. Elle est connectée, au travers des hybrides, au récepteur et à l'émetteur qui utilisent ainsi la même antenne. Un diagramme de rayonnement quasi isotropique et une très bonne adaptation de l'antenne à l'hybride sont nécessaires pour découpler le récepteur très sensible de l'émetteur.

2.3. Sous-système D'ATtitude. - 2.3.1. Mesures d'attitude. - La détermination de l'attitude de l'axe de rotation par rapport à des axes inertiels est obtenue avec la précision de $\pm 2^{\circ}$ par l'utilisation conjointe d'un senseur d'aspect solaire et d'un senseur infrarouge ou d'albedo suivant les possibilités de détection de la terre.

L'angle de rotation et les impulsions nécessaires au contrôle d'attitude du satellite sont fournis par des senseurs solaires auxiliaires (" gating ») distribués uniformément à la périphérie du satellite.

Le boîtier K1, « électronique de mesure d'attitude», 
effectue le traitement des informations fournies par le senseur d'albedo et le senseur infrarouge. Ce boîtier fournit également les informations concernant l'angle de rotation du satellite et la période de rotation.

2.3.2. Contrôle d'attitude. — Le contrôle d'attitude se fait via le boîtier K2, dénommé « électronique de contrôle d'attitude ». Il remplit les diverses fonctions suivantes :

- il transmet à l'encodeur de télémesure les informations digitales fournies par le senseur solaire,

- il permet le contrôle automatique de l'angle d'aspect solaire (la chaîne de contrôle d'attitude peut être ouverte au départ de ce boîtier),

- il fournit les impulsions électriques nécessaires aux tuyères de réorientation,

- il fournit les impulsions permettant de rattraper la vitesse de rotation du satellite (accélération ou décélération).

2.4. Divers. - Au $\S 7$, il sera également fait mention des boîtiers :

M1 : électronique tampon de télémesure;

D1 : électronique de l'expérience S58 embarquée à bord du satellite.

3. Le problème de propreté magnétique. HEOS-A1 emporte une expérience S24A destinée à mesurer le champ magnétique interplanétaire à l'extérieur de la magnétosphère et dans la zone de transition.

Par suite des très faibles champs magnétiques rencontrés dans l'espace, la perturbation introduite dans la mesure par le satellite lui-même doit être aussi faible que possible et ne peut excéder $1 \gamma$ à l'endroit du magnétomètre (distant approximativement de $2 \mathrm{~m}$ du centre de gravité du satellite).

3.1. Minimiser LA Perturbation magnétique introduite PAR LE SATEllite. - Depuis le début du projet HEOS-A1, un effort tout particulier a été entrepris en ce qui concerne la propreté magnétique du satellite complet. Le programme magnétique mis sur pied à cet effet couvre tous les niveaux du projet et plus particulièrement :

- la sélection des matériaux,

- l'outillage spécial pour la fabrication (usinage et assemblage),

- la sélection des composants et pièces détachées du point de vue de la propreté magnétique,

- la réduction au strict minimum des matériaux magnétiques à utiliser inévitablement,

- la conception soignée de tous les circuits électriques pour éviter les champs magnétiques dus à la circulation de courant.

3.2. Mesures magnétiques. - Un programme de mesures intensives a été mis sur pied au niveau système et sous-système.

En vue de réaliser le niveau de confiance maximum dans les résultats magnétiques du satellite HEOS-A1, toutes les pièces sont mesurées avant assemblage. Les champs maxima tolérés pour tout ensemble embarqué (système et sous-système) sont définis par des spécifications et ces mesures magnétiques constituaient une part importante de la qualification de la conception.
La procédure de mesure suivante a été utilisée afin d'éliminer l'effet de champs permanents initiaux incontrôlés et variables et conduire à des résultats reproductibles lors des essais :

- Un système d'axes orthogonaux de l'objet testé est repéré par rapport à une monture utilisée dans la station d'essais magnétiques.

- On enregistre le champ magnétique avant et après rotation de $90^{\circ}$ de l'objet afin de déterminer le champ magnétique permanent initial en valeur et en direction. Ces mesures sont refaites après exposition dans un champ magnétique continu de $25 \mathrm{G}$ et un traitement de démagnétisation dans un champ magnétique alternatif de $50 \mathrm{G}$ selon chacun des axes de l'objet.

- Afin de supprimer le champ induit par le champ magnétique terrestre, l'objet est placé à l'intérieur d'un système de bobine triaxiale de "Helmholtz » qui annule le champ magnétique terrestre à l'endroit de la mesure.

Tous les sous-ensembles contenant des circuits électriques sont également mesurés pour fournir les informations sur les champs magnétiques dus à une circulation des courants dans la bande allant du continu à $28 \mathrm{kHz}$. La distance de mesure (en général $45 \mathrm{~cm}$ pour les boîtiers) est ajustée en fonction de la dimension de l'objet et du niveau du champ magnétique relevé lors d'une mesure préliminaire.

3.3. Compensation magnétique. - La compensation de champ magnétique est possible si les champs résiduels sont stables dans les conditions d'environnement envisagées.

Autant que possible, les champs rayonnés par les courants sont compensés au niveau du boîtier pour éviter des champs excessifs durant la commutation de l'équipement.

En particulier, l'implantation est étudiée pour obtenir la meilleure compensation possible des moments magnétiques individuels.

4. Les moyens de mesures magnétiques. - 4.1. Le champ magnétique rayonné par un objet à une certaine distance se compose principalement d'un champ dit « permanent » et d'un champ induit - en fait, induit par l'objet auquel est appliqué un champ extérieur (en particulier le champ magnétique terrestre). Ce champ induit est, en première approximation, vectoriellement proportionnel au champ extérieur appliqué (pour des champs relativement faibles); le champ permanent est le champ mesuré en l'absence de tout champ extérieur (champ magnétique terrestre) appliqué à l'objet.

Le champ magnétique interplanétaire étant de l'ordre de $50 \gamma$ (en tous les cas très faible), le champ magnétique résiduel dû au satellite à l'endroit du magnétomètre de mesure $\mathrm{S} 24 \mathrm{~A} \alpha$ sera en ordre principal un champ permanent. Par conséquent, toutes les méthodes de mesure et moyens d'essais viseront essentiellement à déterminer le champ permanent au niveau des sous-ensembles. Accessoirement, on déterminera le champ induit : on aura ainsi une idée grossière sur l'importance des matériaux ferromagnétiques doux présents dans le sous-ensemble.

Tous les moyens d'essais doivent permettre la séparation du champ permanent (ou « perm») du champ 
induit (ou « induit »), ceci conduit à deux grandes catégories de moyens d'essais :

a) On trouve, d'une part, les appareillages pour lesquels l'échantillon est placé en champ nul au sein du système de mesure, de telle sorte qu'on élimine le champ induit.

b) Et, d'autre part, les appareillages dans lesquels l'échantillon à mesurer est mis en mouvement (le plus souvent en rotation), le champ permanent suit le mouvement de l'échantillon tandis que l'induit reste plus ou moins aligné sur le champ extérieur. Dans cette catégorie d'équipement, on trouve les « spinner » lorsque l'échantillon est mis en rotation rapide dans le champ terrestre.

Par ailleurs, les procédures d'essais ont mis à profit les caractéristiques de l'échantillon en translation ou en rotation (signature circulaire) par rapport au dispositif de mesure, et ce en champ nul ou non.

4.2. - En janvier 1966, alors que le projet HEOS-A1 était dans sa phase de conception, simultanément les premières investigations du point de vue des moyens d'essais étaient faites à E.T.G.A.

E.T.C.A. devait disposer, fin de l'année 1966, d'une station de mesures magnétiques, capables de fonctionner dans un environnement industriel.

Par un hasard heureux, le Centre de Physique du Globe à Dourbes (en Belgique), hautement spécialisé dans les études de géomagnétisme, lançait également des études dans le domaine du magnétisme des roches.

Ce problème n'était pas sans présenter d'étroites similitudes avec celui de propreté magnétique des sous-ensembles HEOS-A1, et ce spécialement du point de vue des méthodes de mesure et des moyens d'essais. C'est pourquoi, tout au long du projet, le Centre de Physique à Dourbes et E.T.C.A. ont travaillé en étroite collaboration.

En résumé, la section chargée des mesures magnétiques à E.T.C.A. s'est appuyée sur :

- une station capable d'effectuer des mesures en environnement industriel (à E.T.C.A.),

- l'ensemble des moyens d'essais progressivement disponibles à Dourbes au long du projet.

4.3. Les moyens D'essais utilisés a E.T.C.A. AU NIVEAu Sous-ENSEmble. - Outre un ensemble de sondes magnétiques Forster (de sensibilité $0,1 \gamma$ et d'un détecteur d'impuretés (gradiomètre) Schonstedt MID1 Héliflux), E.T.C.A. dispose d'une installation de mesure magnétique de sous-ensembles où le champ magnétique terrestre est annulé par un système triaxial de bobines de Helmholtz et capable de fonctionner en environnement industriel. La mesure proprement dite se fait à l'aide de magnétomètres à aimant (type « Jolivet ») montés en différentiel pour l'élimination des variations du champ magnétique terrestre dans la mesure. L'installation est équipée d'une chaîne de mesure verticale et horizontale.

Gette installation a été livrée sur spécifications par la Compagnie des Compteurs (Paris-Montrouge) sous l'étiquette EMM-2-20-2000. Les figures 3 et 4 donnent un aperçu général de cette installation.

Pour compléter cette installation de mesure, des moyens d'essais pour magnétiser et démagnétiser (cf. $\S 5$ : Spécifications et procédures) étaient nécessaires.

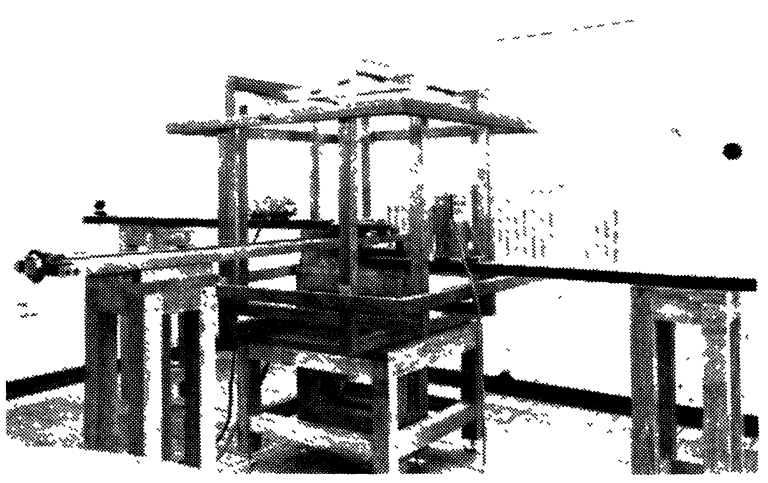

FIG. 3. - Installation EMM-2-20-2000. Bobines de Helmholtz pour la compensation du champ magnétique terrestre et magnétomètres de mesure (de référence) pour les chaînes horizontale et verticale.

Caractéristiques de l'installation EMM-2-20-2000 : Bobines de Helmholtz : $121 \times 121 \mathrm{~cm}$ (pour la plus grande); uniformité du champ : $1 \%$ dans une sphère de $10 \mathrm{~cm}$ de rayon, $2 \%$ dans une sphère de $15 \mathrm{~cm}$ de rayon; champ résiduel au centre des bobines : il n'était pas demandé une compensation meilleure que $100 \gamma$; précision des magnétomètres : $0,05 \gamma$; précision des mesures : au moins $0,1 \gamma$; bande passante ( $12 \mathrm{~dB}$,'octave) de l'installation complète (du point de vue de la mesure) choisie par commutation : $15 \mathrm{~Hz}$, pratiquement inutilisable dans l'environnement de l'installation; $2 \mathrm{~Hz}$, la seule utilisée tout au long du projet; six gammes de mesure; réglage $\mathrm{du}$ " zéro " indépendant sur chaque onde : $\pm 50 \mathrm{\gamma}$; enregistrement : deux pistes (mesures " horizontale " et " verticale ").

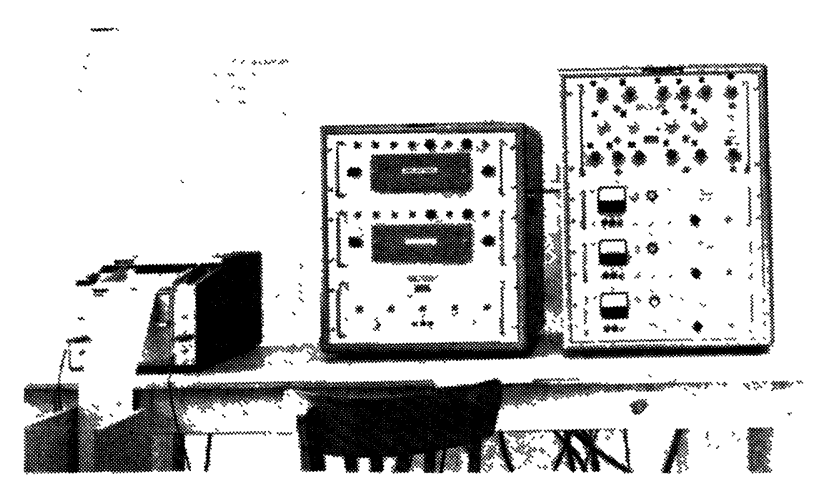

FIG. 4. - Installation EMM-2-20-2000.

Baies électroniques de contrôle et de mesure.

La figure 5 donne un aperçu général de cette installation, qui a été montée de telle sorte à permettre la démagnétisation de l'échantillon de manière automatique en un laps de temps prédéterminé; en outre, le processus de démagnétisation peut s'effectuer en mettant l'échantillon en mouvement dans un champ alternatif décroissant (processus de démagnétisation par « tumbling ») ou bien en maintenant l'échantillon fixe dans un champ alternatif décroissant, mais en ayant compensé le champ magnétique terrestre. 


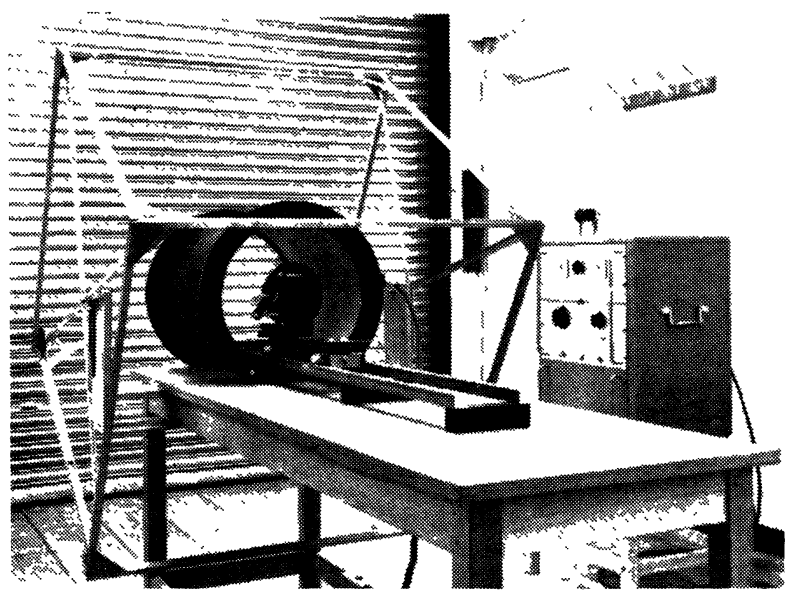

FIG. 5. - Installation de magnétisation-démagnétisation mise en œuvre à E.T.C.A.

Alimentation : alternatif $50 \mathrm{~Hz}$; champ continu de magnétisation (réglable) maximum 200 Oe ; champ alternatif de démagnétisation (réglable) 200 Oe max. ; champ continu superposable au champ alternatif de démagnétisation 2 Oe max. ; dimensions du solénoïde, longueur : $80 \mathrm{~cm}$; diamètre : $45 \mathrm{~cm}$.

4.4. Les moyens D'essais disponibles au Centre de Physique du Globe a Dourbes pendant le projet HEOS-A1. - Ce Centre de Physique du Globe est situé dans un endroit idéalement choisi pour effectuer des mesures magnétiques. Depuis 1948, le géomagnétisme est une des activités principales du Centre.

Outre l'appareillage de mesure classique dans un centre de géophysique, le Centre s'est équipé de moyens de mesure modernes pour l'enregistrement automatique du champ magnétique terrestre et la

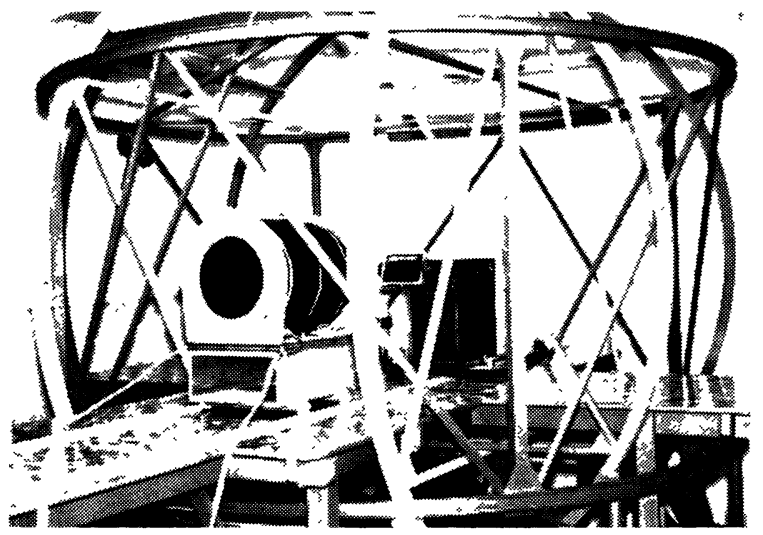

FIG. 6. - Dans cette installation, l'échantillon est maintenu en champ nul à l'intérieur de bobines circulaires triaxiales (environ $2,3 \mathrm{~m}$ de diamètre). La compensation du champ terrestre s'effectue automatiquement à l'aide de petites bobines circulaires homothétiques aux bobines de compensation, elles sont placées à l'extérieur de l'installation de mesure. La mesure s'effectue à l'aide de sondes Forster (précision $0,5 \gamma$ ).

En outre, il est possible d'appliquer des traitements de démagnétisation avec des champs réglables en intensités (maximum $1000 \mathrm{G}(\mathrm{AC})$ ) en mettant simultanément l'échantillon en mouvement (démagnétisation par " tumbling ") ou non. détection précise de moments magnétiques permanents d'échantillons de roches.

A titre d'exemple, disons que sur la superficie du Centre diverses sondes magnétiques (magnétomètres

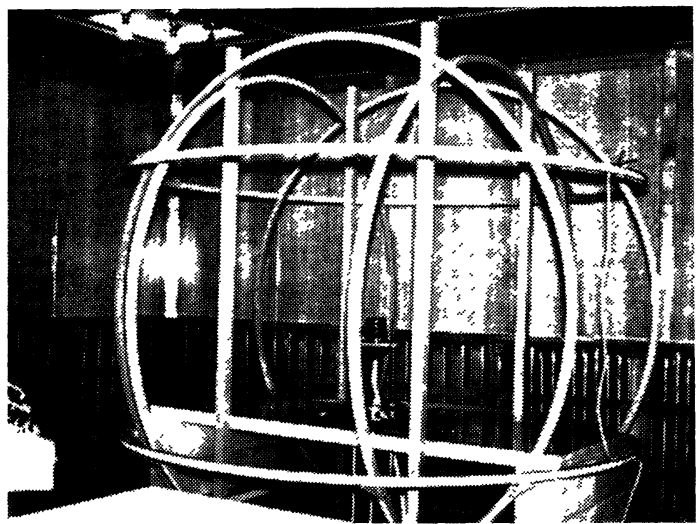

FIG. 7. - Appareil Dycome-Vickers : cet appareil est bien connu sur le marché. Il a servi principalement à des étalonnages de magnétomètres.

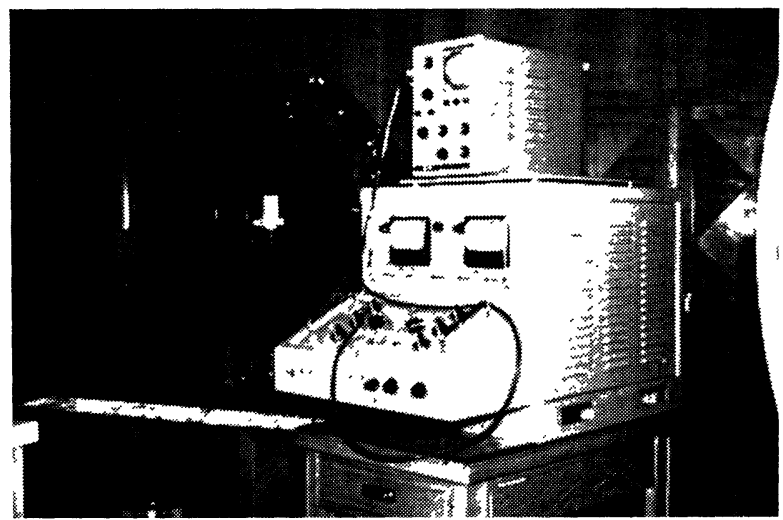

FIG. 8. - Inductomètre "Thellier " : cet appareil du type "spinner " conçu par le Professeur Thellier (de la Faculté des Sciences à Paris) a été industrialisé par la Société Sercel.

Après réglages et compensations, on peut lire directement la valeur du moment magnétique permanent ou de la susceptibilité magnétique.

à pompage optique sur le rubidium - marque Varian) fournissent des informations enregistrées sur bandes magnétiques, lesquelles sont traitées ensuite sur calculateur digital. Il n'est pas du ressort de cet article de faire l'inventaire descriptif des moyens d'essais dont dispose le Centre de Physique du Globe; on se bornera à citer sommairement les quelques installations utilisées au cours du projet HEOS-A1.

Signalons enfin que le Centre de Dourbes dispose d'un magnétomètre vibrant EMR, d'un « spinner » PAR (Princeton Applied Research) et d'un ensemble de magnétomètres à vapeur de rubidium Varian, type V4938, dont deux sont utilisés en permanence sur le Centre pour l'enregistrement du champ magnétique terrestre sur une unité centrale. Les sondes Varian de réserve ont été utilisées pour les essais magnétiques effectués sur la botte électrique du modèle M1 du satellite HEOS-A1 (cf. $\S 7.2 .7$ ). 
5. Spécifications et procédures (calibration). La figure 9 ci-après, extraite de la spécification $\mathrm{n}^{0}$ 301.SP.00.103 dénommée « HEOS-A Satellite Magnetic Cleanliness Specification » et d'application

Design Goal

MAXIMUM MAGNETIC FIELD DISTURBANCE (Gamma's)

\section{Test Condition}

1. Initial perm

2. Post 25 G exposure

3. Post 50 G

4. DG stray field :

5. Perm field after power $\mathrm{ON} / \mathrm{OFF}$

6. AC stray field : frequency range :

$\begin{array}{rlrl}1-20 & \mathrm{kcps} & 200 & 25 \\ 20-22 & \mathrm{kcps} & 80 & 10 \\ 22-26 \mathrm{kcps} & 1 & 0.125 \\ 26-28 \mathrm{kcps} & 80 & 10\end{array}$

$\left(^{*}\right)$ All distances are measured from the center of gravity of the box.

Extrait de la spécification SPEC. n 301 .SP.00.103 (Rev. 1) " HEOS-A Satellite Magnetic Cleanliness Specification ".

FIG. 9. - Design Goal assign to Subassemblies.

dans le cadre du projet HEOS-A, donne un aperçu des buts que l'on s'était proposé d'atteindre pour chaque sous-ensemble et les boîtiers en particulier.

Buts à atteindre bien plus que spécifications, au niveau sous-ensemble car ces « guides » ne tiennent pas compte de la physionomie particulière de chacun de ces sous-ensembles. Et seul l'effet global résultant de l'interaction de chacun des sous-ensembles à l'endroit du magnétomètre $\mathrm{S} 24 \mathrm{~A} \alpha$ constituait la spécification à respecter.

Afin de pouvoir comparer les résultats de mesures des divers moyens d'essais magnétiques, les étalonnages s'effectuaient à l'aide de trois aimants de calibration (dont la stabilité était garantie) couvrant trois gammes de mesure des appareils. Ces aimants avaient été mesurés avec précision à G.S.F.C. et au Centre de Physique du Globe de Dourbes (ainsi que dans divers autres centres de géophysique en Europe). L'étalonnage des gammes intermédiaires se faisait à l'aide d'une bobine dans laquelle circulaient des courants prédéterminés.

A partir du prototype $\mathrm{P} 1$, les mesures ont été effectuées en suivant une procédure rigoureuse et suivie dans toutes les sections magnétiques du projet.

6. Les essais sur composants (pièces détachées). De nombreux essais ont été effectués sur les composants. Parmi les pièces détachées envisagées, on a trouvé en ordre principal les relais (Babcock ou Filtors), les microcircuits (principalement de la série Texas), des noyaux magnétiques pour les convertisseurs basse tension, des résistances, des capacités, certains matériaux, pour lesquels il serait trop laborieux de rapporter ici les résultats d'essais dans le détail.

Il est cependant nécessaire de dire qu'en début de projet on croyait nécessaire d'effectuer un contrôle à $100 \%$ sur l'ensemble des pièces détachées.

Le projet se déroulant, des contraintes particulières se sont présentées en ce qui concerne le choix des pièces détachées, contraintes parmi lesquelles on a retrouvé principalement le problème des pièces à haute fiabilité («HI REL parts »). Une autre contrainte qui s'est avérée d'importance dans le projet était le temps alloué aux mesures magnétiques sur pièces détachées en relation avec le planning général de chacun des modèles du projet. Finalement, on s'est contenté d'un échantillonnage raisonnable des pièces détachées caractéristiques à inclure dans chacun des boîtiers.

Des $100 \%$ des mesures magnétiques sur l'ensemble des pièces détachées estimés nécessaires en début du projet, on en arrivait en fin de projet à des mesures magnétiques sur $10 \%$ de l'ensemble des pièces détachées intervenant dans les boîtiers comme échantillon le plus caractéristique sur les pièces détachées livrées par les fournisseurs.

Pour des raisons impérieuses de propreté magnétique, certaines pièces détachées ont dû faire l'objet de mesures approfondies à $100 \%$ comme par exemple les relais Filtors à inclure dans la boîte de jonction N1 ou encore les noyaux magnétiques bobinés intervenant dans le boîtier convertisseur basse tension N3. Lors de ces essais, on a pu constater par ailleurs que lors de la mise en œuvre des composants, un gain substantiel du point de vue propreté magnétique pouvait être aisément obtenu en coupant les fils de connexion de la pièce détachée : principalement les transistors, résistances et capacités, et en faisant subir ensuite aux composants un traitement de démagnétisation, dans un champ de $50 \mathrm{G}$ alternatif, et ceci pour éliminer l'effet de magnétisation introduit par l'outil lors de la coupe.

Enfin, des mesures particulières ont été effectuées sur des échantillons de matériau, métaux à utiliser pour la construction et l'assemblage des boîtiers, divers types de mousses destinés à constituer les pottings.

Ces mesures ont facilité la mise en œuvre technologique au niveau de chacun des boîtiers.

\section{Essais sur les sous-ensembles électriques du} satellite. - 7.1. Articulation DU MAGNÉTisme DANS LE GADRE DU PROJET HEOS-A1. - Le programme du projet comprenait la réalisation de quatre modèles :

- une maquette M1 : principalement destinée à l'étude,

- une maquette M2 : destinée aux essais d'intégration électrique et d'interface,

- un prototype $\mathrm{P} 1$ : destiné aux essais d'intégration électrique et d'environnement,

- deux exemplaires de vol F1, F2.

Comme M2 était une maquette (au demeurant fort proche, dans sa configuration, des exemplaires de vol), certaines déviations dans la mise en œuvre ont été admises, par exemple les connecteurs n'étaient pas non magnétiques, les composants utilisés n'étaient pas du type HI-REL, etc.

Dès le début du projet, la propreté magnétique a été pensée comme intégrée au contexte général de 
l'assurance, du contrôle de qualité et de la fiabilité des sous-ensembles. Cette intégration est devenue effective lors des séquences d'essais d'acceptance des exemplaires de vol.

7.2. Revue DES BOITIERS ET DES RÉSUltats D'ESSAIS MAGNÉtiques. - 7.2.1. CoMmentaire SUR LES VALEURS DE GHAMPS MAGNÉTIQUES RELEVÉES SUR LE BO1̂TIER N1 (voir tableau I). - 1. Aucun essai magnétique (en champ continu ou alternatif) n'a été exécuté en stimulant le boîtier par des courants externes. En effet :

- Les relais utilisés sont tous du type à verrouillage magnétique et la stimulation de ceux-ci par télécommande est toujours brève. En opération, on ne doit normalement détecter aucun champ magnétique alternatif.

- Ce boîtier, de par sa structure et sa relation avec tous les équipements de bord, était à considérer dans le contexte d'intégration du satellite complet.

Finalement, seuls les champs magnétiques statiques offraient un intérêt, principalement ceux correspondant aux relais en position normale d'utilisation. Le tableau II donne quelques valeurs de champs relevées par signature circulaire pour divers états de basculement des relais.

2. Le tableau I donne quelques informations sur les résultats d'essais effectués sur les divers modèles élaborés au long du projet.

Bô̂tier N1 : Bô̂te de jonction (sous-ensemble d'alimentation).

\section{TABLEAU I}

Champs magnétiques Relevés a $45 \mathrm{~cm}$ DU GENTRE DE GRAVITÉ DU BOITIER

$\begin{array}{cccc} & \text { PERM } & \text { DEPERM } & \\ \text { MODÈLE } & 25 \mathrm{G}(\mathrm{DC}) & 50 \mathrm{G}(\mathrm{AC}) & \text { REMARQUE } \\ - & - & - & - \\ \text { M2 } & 61,9 & 58 & \text { Relais Babcock } \\ \text { P1 } & 36,2 & 36 & \text { " } \\ \mathrm{P}^{\prime} 1 & 19 & 5,5 & \text { Relais Filtors } \\ \text { F1 } & 21 & 4,4 & " \\ \text { F2 } & 5,9 & 4,1 & "\end{array}$

1. Toutes les valeurs de champ sont exprimées en gamma.

2. Tous les relais sont en position d'utilisation lorsque le satellite est opérationnel.

\section{TABLEAU II}

\begin{tabular}{|c|c|c|}
\hline \multirow{3}{*}{$\begin{array}{c}\text { RELAY POSITION } \\
\text { ALL RELAYS } \\
\text { ON BESIDE }\end{array}$} & \multicolumn{2}{|c|}{ Gircular } \\
\hline & \multicolumn{2}{|c|}{ SIGNATURE $p-p$ VALUE } \\
\hline & $X-Y$ & $X-Z$ \\
\hline- & - & - \\
\hline S24A-OFF & 4 & 5 \\
\hline S24B-OFF & 5 & 4,3 \\
\hline S24C-OFF & 9,9 & 4,8 \\
\hline S72-OFF & 7,8 & 7 \\
\hline S73/58-OFF & 8,4 & 5 \\
\hline S79-OFF & 6 & 5,5 \\
\hline IR1-ON & 3,8 & 4,8 \\
\hline IR2-ON & 5,4 & 5,8 \\
\hline All relays "OFF & & \\
\hline Albedo "ON" & 7,2 & 6,2 \\
\hline
\end{tabular}

REVUE DE PHYSTQUe APPLIQUÉE. - T. 5. No 1 . FÉVRIER 1970.
Ce boîtier contient environ 16 relais.

Modèle M2. - Les relais Babcock BR17A étaient utilisés. Ils étaient montés par paires se compensant, en principe, mutuellement. A ce stade, on s'est rendu compte que les paires devraient être judicieusement sélectionnées, et qu'un nombre pair de relais était strictement nécessaire (ce modèle contenait un nombre impair de relais).

Modèle P1. - Les relais préalablement choisis étaient montés en paires sélectionnées. Le boîtier contenait cette fois un nombre « pair de paires de relais ».

Néanmoins, à ce stade, il a fallu admettre que la dispersion des champs magnétiques relevés sur les relais Babcock était trop grande et qu'en outre un niveau magnétique moyen plus faible serait bien utile. Par ailleurs, les deux états de basculement d'un relais étaient fortement différenciés du point de vue magnétique.

On s'est tourné alors vers les relais Filtors «Blue Ribbon » qui avaient un niveau magnétique plus faible, une moindre dispersion, et moins différentiés quant à l'état de basculement.

Modèle P1' (cf. fig. 10). - Les relais Filtors ont été triés, pairés soigneusement, et les paires finalement utilisées ont été choisies parmi un lot. L'amélioration obtenue était sensible sans cependant que l'on puisse atteindre la spécification de $2 \gamma$ pour l'état après démagnétisation à $50 \mathrm{G}(\mathrm{AC})(50 \mathrm{~Hz})$.

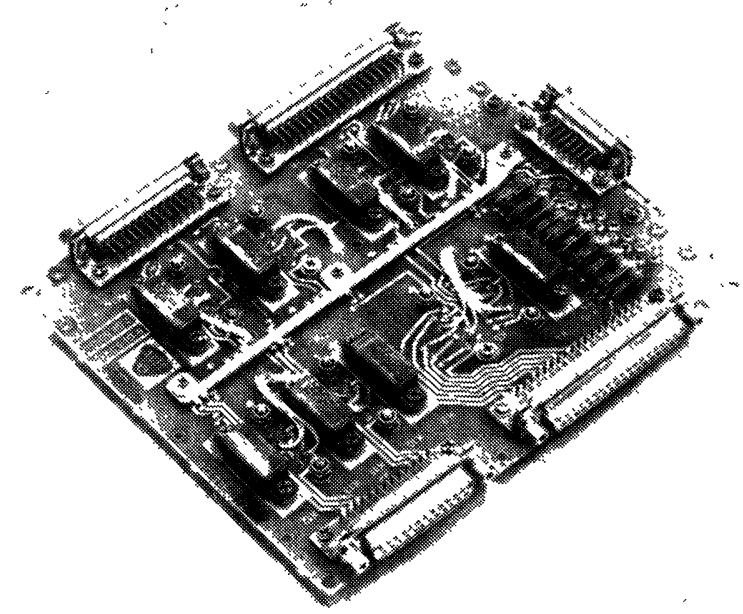

FIG. 10. - Plaquette à relais de la boîte de jonction N1.

Les relais sont montés de part et d'autre de la plaquette.

Modèles F1/F2. - Les relais utilisés provenaient d'une fabrication spéciale présentant un minimum de dispersion quant aux champs magnétiques rayonnés. Ils avaient été approvisionnés en nombre suffisant pour faire une sélection tant au niveau de la pièce que de la paire et des quads.

Une procédure, relativement souple, a été mise en œuvre pour permettre, lors d'essais préliminaires à la fabrication des exemplaires de vol, de s'assurer que les champs magnétiques réellement les plus faibles étaient obtenus. Malgré cela, il n'a pas été possible de descendre en dessous de $2 \gamma$.

Il semble, d'ailleurs, à la suite d'essais auxiliaires 
faits en d'autres configurations, que cela soit physiquement difficile, compte tenu de la nécessité d'utiliser 16 relais.

7.2.2. Commentaire sur les Valeurs De Ghamps MAGNÉTIQUES RELEVÉES SUR LE BOITIER N2 (tableau III). - 1. Caractéristiques du boîtier. - Ce boîtier contient un nombre important de pièces détachées conventionnelles, une paire de relais de commutation pairés, et un ensemble de transistors de puissance.

2. Pour chaque modèle, le câblage des circuits de puissance était soigneusement vérifié afin de compenser au mieux les champs magnétiques ("Stray »), produits par la circulation des courants - raccordement par fils torsadés et surfaces de boucles réduites au strict minimum ( fig. 11).

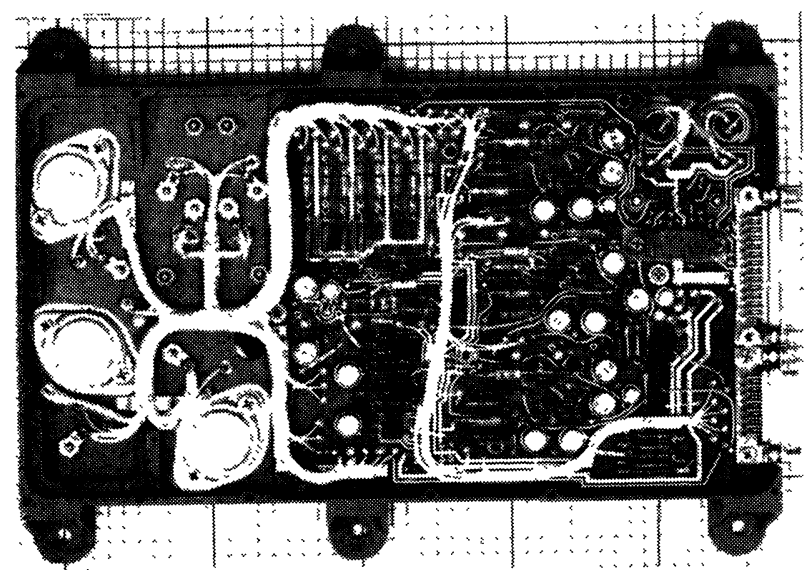

FIG. 11. - Le régulateur de tension N2. Vue montrant le câblage du boîtier (bottes torsadées) (modèle P1 avant enrobage).

L'unique relais Babcock BR17A sur le modèle M2 (cf. tableau III, Remarque 1) a été remplacé par une paire compensée de relais (tableau III, Remarque 2). Il en a été de même pour le modèle $\mathrm{P} 1$, on a cependant davantage veillé à la compensation des courants. Ultérieurement, sur le même modèle $\mathrm{P} 1$, la paire de relais Babcock a été remplacée par une paire compensée de relais Filtors (cf. tableau III, Remarque 3), laquelle a fait l'objet d'une sélection plus soignée pour les modèles de vol F1 et F2.

Aucun champ magnétique alternatif n'a été détecté lors des mesures faites sur les divers modèles.

7.2.3. Commentaire sur les valeurs de Ghamps MAGNÉTIQUES RELEVÉS SUR LE BOITIER N3 (tableau IV). - 1. Caractéristiques. - Ce boîtier comprend deux convertisseurs identiques. Ceux-ci sont construits en éléments conventionnels : transistors, résistances, condensateurs, transformateurs toriques adéquatement bobinés, self-compensés, etc. (fig. 12).

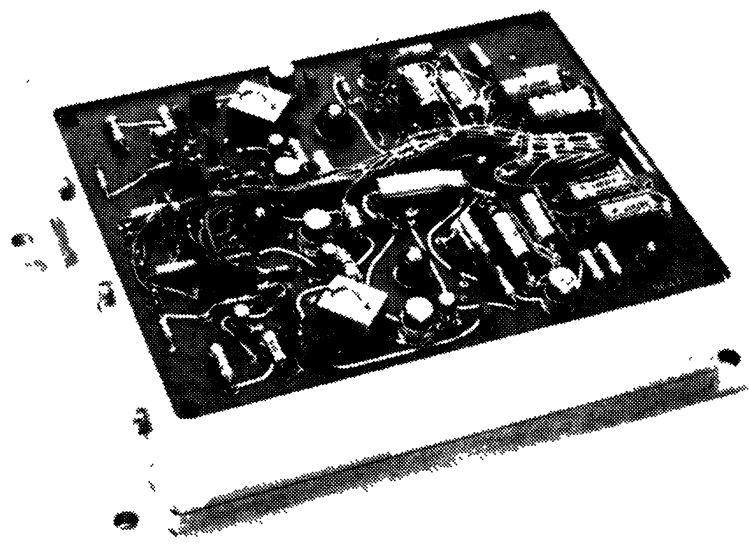

FIG. 12. - Convertisseurs basse tension (vue avant enrobage).

2. Les difficultés se situaient dans les champs magnétiques rayonnés par les courants au niveau des transformateurs avant redressement. Ce problème avait été pressenti mais sous-évalué, ainsi que le montre la valeur de $91,5 \gamma$ relevée sur le modèle M2 (tableau IV). Les bobinages des transformateurs du circuit $3,5 \mathrm{~V}$ de puissance ont été mieux étudiés et une procédure d'assurance de la qualité a été élaborée pour chaque transformateur avant assemblage dans le boîtier. L'amélioration obtenue sur le modèle P1 a été substantielle sans pour autant respecter la spécification de $2 \gamma$ mise sur ce poste-là.

Bô̂tier N2 : Régulateur de tension (sous-ensemble d'alimentation).

TABLEAU III

Champs magnétiques relevés a $45 \mathrm{~cm}$ du Gentre de Gravité du boîtier

$\begin{array}{ccccc}\text { Perm } & \text { Deperm } & & \\ \text { Modìle } & 25 \mathrm{G}(\mathrm{DG}) & 50 \mathrm{G}(\mathrm{AC}) & \text { Stray } & \text { Remarque } \\ - & - & - & - & - \\ \text { M2 } & 62,5 & 36 & - & (1) \\ \text { M2 } & 43,5 & 8,5 & 2,4 & (2) \\ \text { P1 } & 32 & 9,4 & 1,7 & \\ \text { P1 } & 37,5 & 3,9 & 1,6 & (3) \\ \text { F1 } & 28,8 & 2,5 & 1,9 & \\ \text { F2 } & 30,5 & 1,7 & 1,6 & \end{array}$

Toutes les valeurs de champ sont exprimées en gamma. 
Boittier N3 : Convertisseurs basse tension (sous-ensemble d'alimentation).

\section{TABLEAU IV}

Champs magnétiques globaux Relevés a $45 \mathrm{~cm}$ du Gentre de gRavité du boîtier

\begin{tabular}{|c|c|c|c|c|}
\hline & Perm & Deperm & & \\
\hline MODÈLE & $25 \mathrm{G}(\mathrm{DC})$ & $50 \mathrm{G}(\mathrm{DC})$ & StRAY & REMARQUe \\
\hline - & - & - & - & - \\
\hline M2 & 22,6 & 1,1 & $91,5(*)$ & $\begin{array}{l}\left(^{*}\right) \text { Valeur maximale pour } \\
\text { le convertisseur C1 }\end{array}$ \\
\hline $\mathrm{P} 1$ & 27,5 & 1,7 & $\begin{array}{l}3,7 \\
4,5\end{array}$ & $\begin{array}{ll}\text { Convertisseur } & \mathrm{C} 1 \\
\text { Convertisseur } & \mathrm{C} 2\end{array}$ \\
\hline $\mathrm{F} 1$ & 24 & 5 & $13(* *)$ & $\begin{array}{l}(* *) \text { Transformateurs mal } \\
\text { bobinés }\end{array}$ \\
\hline $\mathrm{F} 2$ & 21 & 2,8 & 4 & \\
\hline
\end{tabular}

Toutes les valeurs de champ sont exprimées en gamma.

Bontier N4:Batterie (sous-ensemble d'alimentation).

\section{TABLEAU V}

Champs magnétiQues Relevés a $45 \mathrm{~cm}$ DU CeNTRE DE GRAVité DU boîtier

\begin{tabular}{|c|c|c|c|c|}
\hline MODÈLE & $\begin{array}{c}\text { PERM } \\
25 \mathrm{G}(\mathrm{DG})\end{array}$ & $\begin{array}{c}\text { DePERM } \\
50 \mathrm{G}(\mathrm{DC})\end{array}$ & StRAY & Remaroue \\
\hline MIODELE & - & - & - & - \\
\hline M1 & 5,9 & $<0,2$ & 4,6 & $\begin{array}{l}\text { Maquette d'étude } \\
\text { fournie à E.T.C.A. }\end{array}$ \\
\hline M2 & $<0,2$ & $<0,2$ & 18,8 & Résultats G.S.F.G. \\
\hline M2 & $<0,2$ & $<0,2$ & 3,6 & $\begin{array}{l}\text { Résultats G.S.F.G. } \\
\text { et E.T.G.A. }\end{array}$ \\
\hline P1 & $<0,2$ & $<0,2$ & 2,2 & G.S.F.C. \\
\hline F1 & - & - & 3,3 & G.S.F.C. \\
\hline F2 & $<0,2$ & $<0,2$ & 2,2 & G.S.F.C. \\
\hline F3 & - & - & 3,3 & $\begin{array}{l}\text { (Réserve supplémen- } \\
\text { taire/G.S.F.G.) }\end{array}$ \\
\hline
\end{tabular}

Toutes les valeurs de champs sont exprimées en gamma.

Courant circulant dans la batterie : $2,5 \mathrm{~A}$.

Il était difficile de compenser davantage : en effet, la compensation des enroulements sur le transformateur torique doit se faire avec un nombre entier de spires. La valeur de $13 \gamma$ (« Stray») relevée sur le modèle $\mathrm{F} 1$ a constitué un incident de parcours dans le contrôle « en ligne » du groupe d'assurance de la qualité par suite de non-respect de la procédure élaborée pour le contrôle des transformateurs. Pour le modèle F2, la procédure a été suivie rigoureusement et a fourni le même résultat que pour le modèle $\mathrm{P} 1$.

7.2.4. Commentaire SUR Les VAleurs DE GHAMPS MAGNÉTIQUES RELEVÉES SUR LE BOÎTIER N4 (tableau V). - 1. Caractéristiques. - Le boîtier contenait dix-neuf cellules argent-cadmium (pour des raisons de propreté magnétique) constituant la batterie. Celle-ci était livrée par la Société Yardney (U.S.A.) (fig. 13).

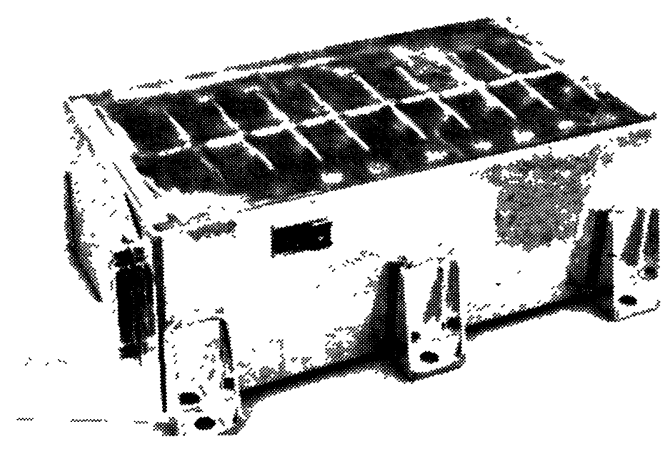

FIG. 13. - Batterie N4 (modèle P1).

2. Les états magnétiques après magnétisation et démagnétisation ne présentaient aucun problème. 
La botte d'interconnexion des cellules a dû faire l'objet d'une étude spéciale, raison même du modèle M1 (maquette d'étude fig. 14). Les cellules fournies par les essais sur ce modèle n'étaient pas absolument

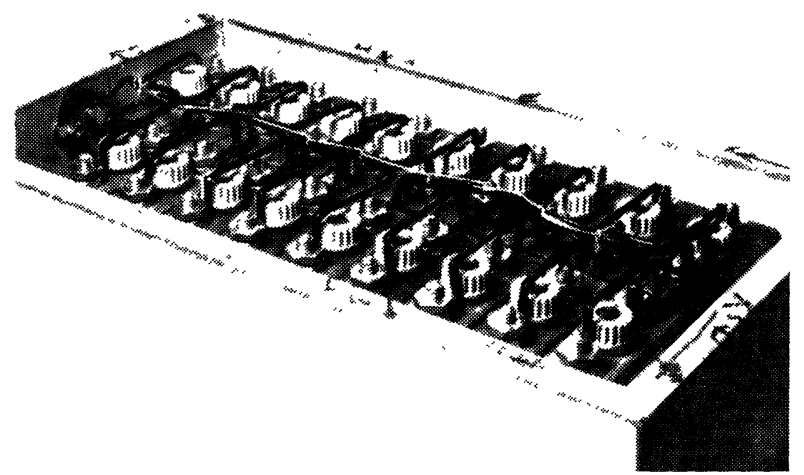

FIG. 14. - Batterie N4 (modèle M1). Vue de la botte.

magnétiquement propres (cf. état Perm 25 G (DG), tableau IV).

Les résultats de l'étude du modèle M1 ont conduit :

- à demander à Yardney d'exécuter la botte, d'effectuer les essais sur le boîtier monté mais non encapsulé et d'ajuster un paramètre déterminé de la botte après essais et enfin d'effectuer les essais finaux d'acceptance à l'aide de la procédure en vigueur dans le cadre du programme HEOS-A1,

- à fournir un gabarit de botte auquel Yardney devait se conformer lors du câblage, toutes les informations et photos dont Yardney pouvait avoir besoin pour la réalisation de la botte.

Les essais magnétiques aux U.S.A. ont été exécutés au «Goddard Space Flight Genter»(G.S.F.C.).

Le courant débité par la batterie lors des essais était de 2,5 A.

Dans un premier temps sur la maquette M2, les instructions n'ont pas été correctement appliquées, d'où le champ important rayonné par la circulation du courant $(18 \gamma)$; dans un second temps, les mesures correctives ont été prises et l'on a retrouvé l'ordre de grandeur attendu ( 3 à $4 \gamma$ ). La conception du câblage a fourni des résultats répétitifs pour les modèles suivants.

7.2.5. Commentaire sur Les Valeurs De Ghamps MAGNÉTIQUES RELEVÉES BOÎTIER N5 (tableau VI) (fig. 15). - Le même commentaire que celui fait pour le boîtier N2 s'applique aussi dans le cas du boîtier N5.

La résistance bobinée «Dale » d'un transistor de puissance s'est trouvée être une source de champ magnétique dû à la circulation de courant (Stray). Un bobinage de compensation a été monté sur le corps de la résistance elle-même pour diminuer cet effet. La vérification de la compensation du champ magnétique se faisant lors des contrôles « en ligne » avant l'assemblage et le montage des sous-ensembles dans le boîtier.

7.2.6. Commentaire SUR Les VAleurs DE GHAMPS MAGNÉTIQUES RELEVÉES SUR LES BOÎTIERS K1，K2, M1, D1 (S58). - Tous ces boîtiers ont un point commun, ils sont constitués d'un empilement important de circuits intégrés Texas SN51, choisis pour leur

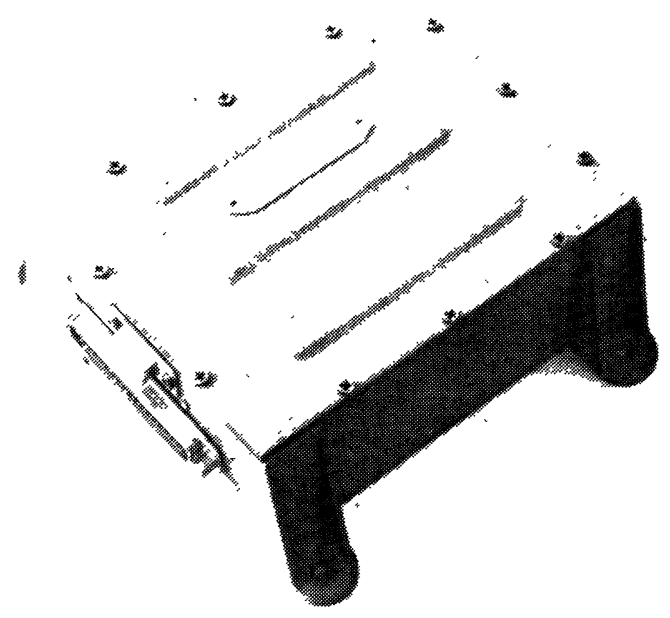

FIG. 15. - Chargeur de batterie N5. Vue du boitier.

propreté magnétique (lors d'essais d'évaluation préliminaires) et leur faible consommation de puissance.

Le champ magnétique le plus important, rayonné par un microcircuit après magnétisation à $25 \mathrm{G}$ (DC), est aligné selon les pattes de celui-ci, ainsi que l'ont montré les essais préliminaires en début de projet. Ce champ pouvait être réduit en recoupant les pattes du microcircuit.

Les modèles M2 et P1 ont mis en évidence que la technique des circuits multicouches utilisés pour atteindre un empaquetage maximum (et un volume minimum) était incompatible avec les microcircuits dont les pattes avaient été recoupées. De surcroît, toutes les pattes de microcircuits étaient alignées selon un axe, pour lequel le champ magnétique maximum (après magnétisation à $25 \mathrm{G}(\mathrm{DC})$ ) était mesuré pour chacun des boîtiers.

A ce stade du projet, il aurait fallu une révision fondamentale du développement de chacun des boîtiers allant à l'encontre des impératifs du planning du moment. D'autant qu'entretemps, les mesures faites sur le satellite complet montraient qu'en l'endroit du magnétomètre $\mathrm{S} 24 \mathrm{~A} \alpha$ les spécifications pour l'ensemble du satellite étaient respectées.

Dans ces conditions, aucune modification n'a été introduite pour les exemplaires de vol; et, jusqu'à la fin du projet, on a relevé des champs importants pour les boîtiers (après magnétisation à $25 \mathrm{G}(\mathrm{DC})$ ).

7.2.7. Commentaire sur les essais magnétiques EFFEGTUÉS SUR LA BOTTE DU MODÈLE M1 DU SATELLITE. - En début de projet, il était apparu extrêmement séduisant durant la phase modèle M1 (phase maquette d'intégration électronique) d'effectuer des essais magnétiques sur la maquette de définition de la botte - quoiqu'elle ne fût pas conçue en vue de cet essai. Un essai préliminaire préparé à E.T.C.A. avait permis de se rendre compte que le champ rayonné par la circulation de courant dans la botte (raccordée à ses terminaisons par des charges fictives simulant la consommation des divers boîtiers) était faible. On s'est tourné alors vers l'utilisation des magnétomètres à vapeur de rubidium (Varian) montés en gradiomètres. Les mesures ont été exécutées au Centre de Physique du Globe à Dourbes (Belgique). L'un des deux magné- 
Bô̂tier N5 : Chargeur de batterie (sous-ensemble de l'alimentation).

\section{TABLEAU VI}

Champs magnétiques Relevés a $45 \mathrm{~cm}$ du Gentre de GRavité du boîtier

$\begin{array}{ccccc}\text { Modèle } & \text { Perm } & \text { Deperm } & & \\ - & 25 \mathrm{G}(\mathrm{DC}) & 50 \mathrm{G}(\mathrm{AC}) & \text { Stray } & \text { Remarque } \\ \text { M2 } & - & - & - & - \\ \text { P1 } & 10,4 & 5 & 2 & \text { Paire de relais compensée } \\ \text { F1 } & 15,1 & 9,4 & 3,7 & \begin{array}{l}\text { Paire de relais compensée } \\ \end{array} \\ & 21,5 & 2,4 & 1,2 & \text { Résistance « Dale » com- } \\ & & & & \text { pensée; paire de relais } \\ \text { F2 } & 20,5 & 2,0 & 1,3 & \text { Filtors }\end{array}$

Toutes les valeurs de champ sont exprimées en gamma.

\section{TABLEAU VII}

Résultats D'essai pour la gompensation de la RÉsistance bobinée « Dale » (boîtier N5)

$\begin{array}{ccccccc}\mathrm{CAS} & +x & -x & +y & -y & +z & -z \\ - & - & - & - & - & - & - \\ \mathrm{a} & +0,4 & -0,2 & -3,2 & +3,1 & - & - \\ \mathrm{b} & 0 & - & +1 & - & - & -\end{array}$

$N=20$ spires

$N=14$ à 15 spires

$<0,05 \simeq 0,05 \simeq 0,05 \quad 0,05$

Par signature

circulaire

$0,05 \quad 0,05$

\section{TABLEAU VIII}

NOMBRE DE GIRGUITS

BoîTIER

K1

$\mathrm{K} 2$

M1

D1
INTÉGRÉS

170

95

70

205

Bô̂tier K1 : Électronique de mesure d'attitude (sous-ensemble d'attitude).

\section{TABLEAU IX}

Ghamps magnétiques Relevés a $45 \mathrm{~cm}$ du Gentre de GRavité du boîtier

$$
\text { PERM DePERM }
$$

Modèle $25 \mathrm{G}(\mathrm{DG}) \quad 50 \mathrm{G}(\mathrm{AG}) \quad$ Stray

RemarQue

$\begin{array}{llcr}- & - & - & - \\ \text { M2 } & 54,5 & 0,5 & <0,1 \\ \text { P1 } & 54 & <0,2 & 0,3 \\ \text { F1 } & 47,5 & 0,35 & 0,2 \\ \text { F2 } & 46,5 & 0,4 & 0,1\end{array}$

Toutes les valeurs de champ sont exprimées en gamma. 
Boîtier K2 : Électronique de contrôle d'attitude (sous-ensemble d'attitude).

\section{TABLEAU $\mathrm{X}$}

Champs magnétiques Relevés a $45 \mathrm{~cm}$ du Gentre de gravité du boîtier

$\begin{array}{ccccc} & \text { Perm } & \text { Deperm } & & \\ \text { Modèle } & 25 \mathrm{G}(\mathrm{DG}) & 50 \mathrm{G}(\mathrm{AG}) & \text { Stray } & \text { Remarque } \\ - & - & - & - & - \\ \text { M2 } & 48 & 0,5 & 0,2 & \text { Perm important dans la } \\ \text { P1 } & 39,6 & 0,9 & 0,2 & \text { direction des pattes } \\ \text { F1 } & 27,5 & 0,8 & <0,3 & \text { des microcircuits } \\ \text { F2 } & 33,8 & 0,9 & 0,2 & \end{array}$

Toutes les valeurs de champ sont exprimées en gamma.

Boîtier M1 : Électronique tampon de télémesure.

TABLEAU XI

Ghamps magnétiques Relevés a $45 \mathrm{~cm}$ du Gentre de gravité du boîtier

\begin{tabular}{|c|c|c|c|c|}
\hline ModèLE & $\begin{array}{c}\text { Perm } \\
25 \text { G (DG) }\end{array}$ & $\begin{array}{c}\text { DEPERM } \\
50 \mathrm{G}(\mathrm{AG})\end{array}$ & Stray & RemarQue \\
\hline $\mathrm{M} 2$ & $\overline{46}$ & $\overline{1,2}$ & $\overline{0,1}$ & Perm important dans la \\
\hline M1 & 25 & 0,1 & - & direction des pattes \\
\hline F1 & 38 & 0,7 & 0,1 & des microcircuits \\
\hline F2 & 32 & 0,8 & 0,3 & \\
\hline
\end{tabular}

Toutes les valeurs de champ sont exprimées en gamma.

Boîtier D1 : Expérience S58 (boîtier électronique D1).

TABLEAU XII

Champs magnétiques Relevés a $45 \mathrm{~cm}$ du centre de gravité du boîtier

$\begin{array}{ccccc} & \text { Perm } & \text { Deperm } & & \\ \text { Modìle } & 25 \mathrm{G}(\mathrm{DG}) & 50 \mathrm{G}(\mathrm{AG}) & \text { Stray } & \text { Remarque } \\ - & - & - & - & - \\ \text { P1 } & 98 & 0,8 & 0,2 & \text { Id. K1, K2, M1 } \\ \text { P2 } & 68 & 0,5 & 0,2 & \\ \text { F1 } & 46 & 1,4 & 0,2 & \\ \text { F2 } & - & - & - & \end{array}$

Toutes les valeurs de champ sont exprimées en gamma.

tomètres est monté à l'endroit du magnétomètre S24A $\alpha$ (voir fig. 16), la base du gradiomètre était de l'ordre d'une centaine de mètres.

Pour parer à toute éventualité, on disposait à tout moment d'un autre ensemble de deux magnétomètres à vapeur de $\mathrm{Rb}$ montés en différentiel.

D'excellentes mesures reproductibles ont été faites avec une précision de $0,01 \gamma$.

Le tableau ci-après donne une idée succincte des

FIG. 16. - Essais magnétiques effectués sur la botte du satellite (modèle M1) à l'aide de magnétomètres à vapeur de rubidium (Varian V4938).

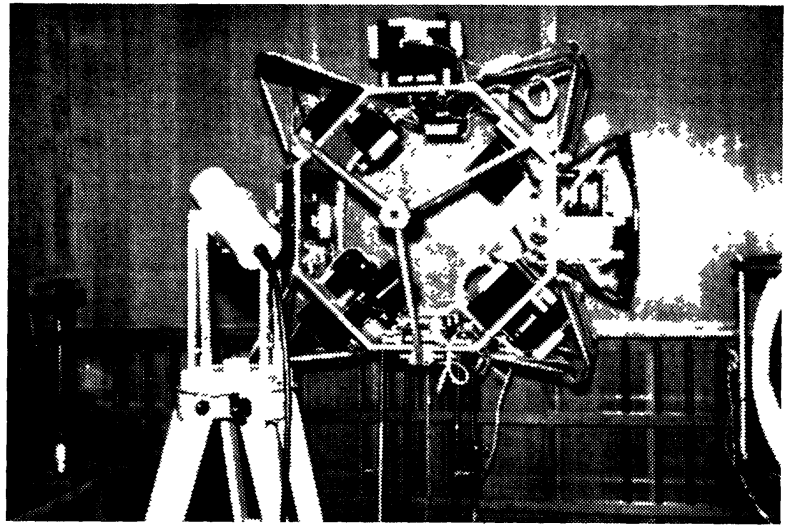


résultats obtenus pour diverses configurations des courants, correspondant à des opérations dites de « jour» et d' " éclipse ». En combinant les composantes des champs magnétiques perpendiculaire et parallèle à l'axe de rotation du satellite, on a relevé les valeurs maximales de champs, reprises ci-après :

$\begin{array}{lc}\text { Mode } & \text { Ghamp total (en gamma) } \\ \text { - } & - \\ \text { Éclipse } & 0,08 \\ \text { Jour } & 0,05 \text { à } 0,072\end{array}$

Un examen plus détaillé des champs a montré qu'une partie importante de la composante de champ perpendiculaire à l'axe de rotation du satellite était due au boîtier simulant la boîte de jonction $\mathrm{N}^{\prime \prime}$ $( \pm 0,04 \gamma)$.

Enfin, la mise en œuvre de la botte sur la maquette du satellite était loin d'être au point :

- certaines ramifications de la botte n'étaient pas bien torsadées,

- il subsistait certaines boucles de courant à proximité des connecteurs de boîtier,

- les charges simulant la consommation n'étaient pas toujours bien compensées.

Les mesures faites sur ce modèle M1 ont constitué davantage un exercice d'orientation et ont permis de prendre les actions nécessaires à la réalisation de la botte (dans une optique de propreté magnétique).

Cet essai mettait bien en évidence combien il était nécessaire d'exécuter rapidement les essais sur le satellite complet avec des boîtiers actifs rayonnant leurs champs magnétiques (dus à la circulation) spécifiques.

Conclusions. - Quoique certains équipements électroniques du matériel banalisé de bord du satellite HEOS-A1 n'aient pu être amenés à rencontrer les spécifications du $\S 5$ (cf. fig. 9), l'ensemble du satellite HEOS-A1 a présenté un champ résiduel total de $0,5 \gamma$ après démagnétisation à $50 \mathrm{G}$ dans un champ alternatif de $50 \mathrm{~Hz}$.

Si certains boîtiers étaient l'objet de limitation physique inhérente du point de vue de la propreté magnétique, d'autres boîtiers sont susceptibles d'amélioration en modifiant l'implantation interne du sousensemble (ceci s'applique en particulier à tous les boîtiers contenant un grand nombre de microcircuits). Ces améliorations pourraient être mises en œuvre à l'occasion de programmes ultérieurs.

Cependant, il semble que, dans tous les cas, un effet de compensation interne intervienne au niveau du satellite complet. Même en tenant compte de cet effet de compensation, il reste toujours souhaitable d'obtenir les champs magnétiques les plus faibles possible au niveau sous-ensembles. 